\title{
Un fantastique petit livre
}

\section{Rouven Porz}

Dr phil., biol. dipl., p.-d., responsable du département Ethique de l'Hôpital de l'lle à Berne, chercheur invité à la VU d'Amsterdam, secrétaire général de la European Association of Centres of Medical Ethics (EACME) et membre de la rédaction Ethique du BMS

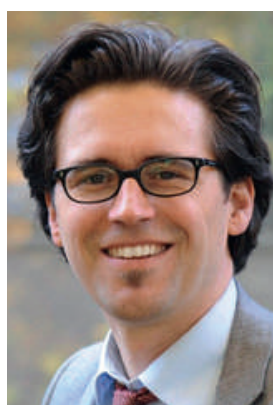

J'ai ma propre liste de questions auxquelles il a été à ce jour répondu de façon fausse ou insuffisante, et que je traîne depuis ces dernières années, voire ces dernières décennies. Comment dort un requin? Qui lit tous les mauvais livres de ce monde? Ou: comment les médecins supportent-ils toute la folie dont ils sont témoins? Une approche de réponse à cette dernière question m'a été fournie par hasard, dans un petit livre fantastique. Avec mes propres mots, la réponse est: souvent ils ne surmontent pas, ils doivent simplement supporter de vivre parfois avec les patients des situations absurdes, qui n'aboutiront à aucune solution rationnelle. Je ne parle pas des décès «normaux», mais des revirements tragico-dramatiques, que personne ne peut (ou ne veut) anticiper.

Vous voyez peut-être très bien ce que je veux dire depuis longtemps déjà, chers lecteurs. Vous êtes tous médecins. Mais pour moi, qui ne le suis pas, ce fut un enrichissement incroyable de découvrir cela dans le merveilleux petit livre du Dr Lukas Fierz, neurologue bernois à la retraite. Le titre: Begegnungen mit dem Leibhaftigen - Reportagen aus der heilen Schweiz ${ }^{1}$. Le Dr Fierz regroupe dans son livre diverses anecdotes très bien écrites sur sa vie de médecin, qui ont toutes

\section{Les 170 pages sont remplies d'exigences normatives implicites et explicites.}

un point commun: elles décrivent des situations et des contextes incroyables qu'il a personnellement vécus dans son travail, et qui lui ont manifestement laissé (comme à moi maintenant) une impression d'absurdité. Je ne vous parlerai pas maintenant des mécaniciens, plongeurs, prostituées, détenus et autres personnes au cœur de ces anecdotes, sous peine de révéler le nœud de ces histoires. Je voudrais simplement souligner quelques réflexions qui m'ont particulièrement intéressé.

Le Dr Fierz parle un langage tout à fait incorrect politiquement. C'est magnifique. Les ambulanciers professionnels s'apparentent à des personnages de Lego, avec leurs uniformes et leurs attitudes, les familles arabes ont en fait encore le droit de payer le mariage en cha- meaux, et les services psychiatriques restent des asiles de fous. Ses histoires sont tellement réalistes que l'on ne peut quasiment plus quitter le livre une fois qu'on l'a commencé.

Un ouvrage passionnant en termes d'éthique médicale. Les 170 pages sont remplies d'exigences normatives

Il plaide pour une culture de la communication avec le patient, qui prenne au sérieux l'univers de ce dernier.

implicites et explicites, d'idées sur comment doit se comporter un médecin, ce qu'il doit faire, quand et comment. Cela m'a beaucoup impressionné. C'est justement cet aveu clair de la réflexion sur sa propre éthique du métier qui me manque souvent dans ma relation avec les médecins (notamment avec les jeunes dois-je avouer). M. Fierz a une idée très précise de là où ses compétences s'arrêtent et de quand il doit solliciter l'aide d'un spécialiste. Il plaide pour une culture de la communication avec le patient, qui prenne au sérieux l'univers de ce dernier. Il prône en fait la curiosité professionnelle (ce sont mes termes, pas les siens). Ses paroles sont approximativement les suivantes: «Dans ces entretiens, il faut être patient. Si l'on a une attitude trop intéressée ou trop curieuse, le courant [avec le patient] ne passe plus. Si l'on pose des questions trop directes, ou que l'on est trop insistant, on rate l'essentiel. Il faut n'écouter qu'à moitié, comme si ce n'était pas important, c'est comme ça que ça marche le mieux. [...] il faut parfois donner la réplique. Ou poser des questions très naïves, qui poussent quelque fois à révéler l'inattendu» (p. 75).

C'est cet inattendu dont il parle qui vous coupe le souffle. Ce drame kafkaïen qui peut se jouer sur la scène de la relation entre médecin et patient, et qui m'a parfois fait un peu peur. Une chose est sûre: après cette lecture, on ne croit plus à la Suisse intacte. Force m'a été de constater, une fois de plus, que le mal existe dans le monde. Merci Dr Fierz, s'il vous plaît n’arrêtez pas d'écrire. 